\title{
Holographic optical element to generate achromatic vortices
}

\author{
Jesús Atencia, ${ }^{1, *}$ María-Victoria Collados, ${ }^{1}$ Manuel Quintanilla, ${ }^{1}$ Julia Marín-Sáez, ${ }^{1}$ \\ and Íñigo J. Sola ${ }^{2}$ \\ ${ }^{I}$ Departamento de Física Aplicada, Instituto de Investigación en Ingeniería de Aragón (I3A), Universidad de \\ Zaragoza, Facultad de Ciencias, Pedro Cerbuna 12, 50009 Zaragoza, Spain \\ ${ }^{2}$ Departamento de Física Aplicada, Universidad de Salamanca, Plaza de la Merced s/n, 37008 Salamanca, Spain \\ "atencia@unizar.es
}

\begin{abstract}
A compound holographic optical element to generate achromatic vortices with high efficiency, based on the combination of two volume phase holograms, is designed and constructed. This element is compact and easy to align. It has high damage threshold, so it can be used with ultraintense laser pulses.
\end{abstract}

(C) 2013 Optical Society of America

OCIS codes: (320.5540) Pulse shaping; (090.1000) Aberration compensation; (090.7330) Volume gratings.

\section{References and links}

1. J. E. Curtis, B. A. Koos, and D. G. Grier, "Dynamic holographic optical tweezers," Opt. Commun. 207(1-6), 169-175 (2002).

2. G. A. Swartzlander, Jr., “Achromatic optical vortex lens," Opt. Lett. 31(13), 2042-2044 (2006).

3. S. Fürhapter, A. Jesacher, S. Bernet, and M. Ritsch-Marte, "Spiral interferometry," Opt. Lett. 30(15), 1953-1955 (2005).

4. G. Molina-Terriza, J. P. Torres, and L. Torner, “Twisted photons,” Nat. Phys. 3(5), 305-310 (2007).

5. M. S. Bigelow, P. Zerom, and R. W. Boyd, "Breakup of ring beams carrying orbital angular momentum in sodium vapor,” Phys. Rev. Lett. 92(8), 083902 (2004).

6. Z. Chen, M. F. Shih, M. Segev, D. W. Wilson, R. E. Muller, and P. D. Maker, "Steady-state vortex-screening solitons formed in biased photorefractive media," Opt. Lett. 22(23), 1751-1753 (1997).

7. M. Fisher, C. Siders, E. Johnson, O. Andrusyak, C. Brown, and M. Richardson, "Control of filamentation for enhancing remote detection with laser induced breakdown spectroscopy,” Proc. SPIE 6219, 621907, 621907-5 (2006).

8. K. Sueda, G. Miyaji, N. Miyanaga, and M. Nakatsuka, "Laguerre-Gaussian beam generated with a multilevel spiral phase plate for high intensity laser pulses," Opt. Express 12(15), 3548-3553 (2004).

9. W. C. Cheong, W. M. Lee, X.-C. Yuan, L.-S. Zhang, K. Oholakia, and H. Wang, "Direct electron-beam writing of continuous spiral phase plates in negative resist with high power efficiency for optical manipulation," Appl. Phys. Lett. 85(23), 5784-5785 (2004).

10. J. W. Sung, H. Hockel, J. D. Brown, and E. G. Johnson, "Development of a two-dimensional phase-grating mask for fabrication of an analog-resist profile," Appl. Opt. 45(1), 33-43 (2006).

11. K. J. Moh, X. C. Yuan, D. Y. Tang, W. C. Cheong, L.-S. Zhang, D. K. Y. Low, X. Peng, H. B. Niu, and Z. Y. Lin, "Generation of femtosecond optical vortices using a single refractive optical element," Appl. Phys. Lett. 88(9), 091103 (2006).

12. G. A. Swartzlander, Jr., “Achromatic optical vortex lens,” Opt. Lett. 31(13), 2042-2044 (2006).

13. V. Y. Bazhenov, M. S. Soskin, and M. V. Vasnetsov, "Screw dislocations in light wavefronts," J. Mod. Opt. 39(5), 985-990 (1992).

14. K. Crabtree, J. A. Davis, and I. Moreno, "Optical processing with vortex-producing lenses," Appl. Opt. 43(6), 1360-1367 (2004).

15. Z. S. Sacks, D. Rozas, and G. A. Swartzlander, Jr., "Holographic formation of optical-vortex filaments," J. Opt. Soc. Am. B 15(8), 2226-2234 (1998).

16. I. G. Mariyenko, J. Strohaber, and C. J. G. J. Uiterwaal, "Creation of optical vortices in femtosecond pulses," Opt. Express 13(19), 7599-7608 (2005)

17. I. J. Sola, V. Collados, L. Plaja, C. Méndez, J. San Román, C. Ruiz, I. Arias, A. Villamarín, J. Atencia, M. Quintanilla, and L. Roso, "High power vortex generation with volume phase holograms and non-linear experiments in gases," Appl. Phys. B 91(1), 115-118 (2008).

18. O. Martínez-Matos, J. A. Rodrigo, M. P. Hernández-Garay, J. G. Izquierdo, R. Weigand, M. L. Calvo, P. Cheben, P. Vaveliuk, and L. Bañares, "Generation of femtosecond paraxial beams with arbitrary spatial distribution,” Opt. Lett. 35(5), 652-654 (2010). 
19. A. Villamarín, J. Atencia, M. V. Collados, and M. Quintanilla, "Characterization of transmission volume holographic gratings recorded in Slavich PFG04 dichromated gelatin plates," Appl. Opt. 48(22), 4348-4353 (2009).

20. A. Villamarín, I. J. Sola, M. V. Collados, J. Atencia, O. Varela, B. Alonso, C. Méndez, J. San Román, I. Arias, L. Roso, and M. Quintanilla, "Compensation of second-order dispersión in femtosecond pulses after filamentation using volumen holographic transmission gratings recorded in dichromated gelatin," Appl. Phys. B 106(1), 135-141 (2012).

\section{Introduction}

During the last years, optical vortex beams have been a key element in the development of some applications, as particle micro-manipulation [1], vortex coronograph [2], interferometry [3] and quantum information [4]. In addition to that, the interest in the non linear propagation of ultraintense and ultrashort vortices [5,6] has been increased, due to its potential applications in some fields, as Laser Induced Breakdown Spectroscopy (LIBS) [7] or phase control in high harmonic generation.

Some of these applications make use of broadband light sources. The challenge is to get the same angular momentum and spatial profile for all the spectral components. So, it is necessary to generate the vortex with an achromatic element or set-up. Besides, when the light source is a laser emitting ultrashort and ultraintense pulses, the elements involved in the vortex generation must have high damage threshold while keeping the width and shape of the pulse undistorted.

There are mainly two techniques to generate optical vortices: spiral phase plates and computer generated holograms (CGHs).

Spiral phase plates are media where the optical path increases with the azimutal angle. They can be obtained by quartz deposition [8], by lithography in resist [9] and photoresist [10] or by engraving in fused silica. With these techniques, high efficiency can be reached and the plates have high damage threshold. The drawback of these plates is the presence of chromatic aberration when they are used with broadband pulses, wider than $40 \mathrm{~nm}$ [11]. Some works have tried to overcome this problem by combining two different materials in the same spiral plate [12], reaching the achromatization of the vortex for two wavelengths, but the achromatization for all wavelengths cannot be obtained.

Computer generated holograms (CGHs) used to generate vortices are gratings that present some dislocation in the diffraction pattern. They are usually amplitude or phase masks recorded in photographic film [13] or generated in LCD [14]. These elements cannot be used directly with high power sources, although it is possible to record a volume phase hologram (VPH) with them, obtaining an element with high efficiency and high damage threshold [15]. CGHs and volume phase holograms are diffractive elements, so they all have chromatic angular dispersion, which make them usefulness with broadband sources. One solution is to combine these elements with refractive elements to compensate the chromatic dispersion and obtain an achromatic vortex [16, 17]. Some authors have proposed an easier solution, with two separated volume and phase gratings [18], but this solution introduce a spatial chirp and, with ultrashort laser pulses, a temporal chirp.

The aim of the present work is to design and construct an element to generate achromatic vortex beams, so it can be used with broadband sources, and with high damage threshold, in order to be used with ultraintense and ultrashort laser pulses. The element is the result of combining two VPHs recorded in commercial dichromated gelatin emulsion Slavich PFG-04. The compound holographic element is compact and easy to align. It can be used with broadband sources, generating a vortex beam with the same angular momentum for all wavelengths, and keeping the spatial and temporal profile when used with ultrashort laser pulses. 


\section{Vortex achromatization with optical holographic elements}

To achieve an element with high efficiency and high damage threshold, a VPH can be recorded with the interference of a plane wave and a vortex beam generated with a CGH. When the volume hologram is illuminated with a broadband plane wave with a Gaussian spatial profile, a set of polychromatic vortices with topological charge $m$ and Gaussian spatial profile emerges of the hologram, each wavelength propagating in different directions.

To compensate the angular chromatic dispersion it is necessary to combine this VPH with a plane volume grating with the same spatial frequency.

We record a volume hologram $\mathrm{H}_{\mathrm{I}}$ with the interference of two plane waves

$$
\begin{aligned}
& U_{r, \mathrm{I}}(x, y)=\mathrm{e}^{-j \frac{2 \pi}{\lambda_{1}} y \sin \alpha_{r}} \\
& U_{o, \mathrm{I}}(x, y)=\mathrm{e}^{-j \frac{2 \pi}{\lambda_{1}} y \sin \alpha_{o}}
\end{aligned}
$$

forming angles $\alpha_{o}$ and $\alpha_{r}$ with $z$ axis (perpendicular to hologram surface).

The intensity pattern resulting from this interference is:

$$
I=\left|\mathrm{e}^{-j \frac{2 \pi}{\lambda_{1}} y \sin \alpha_{r}}+\mathrm{e}^{-j \frac{2 \pi}{\lambda_{1}} y \sin \alpha_{o}}\right|^{2}=2+\mathrm{e}^{-j \frac{2 \pi}{\lambda_{1}} y\left(\sin \alpha_{r}-\sin \alpha_{o}\right)}+\mathrm{e}^{j \frac{2 \pi}{\lambda_{1}} y\left(\sin \alpha_{r}-\sin \alpha_{o}\right)}
$$

The complex transmittance recorded if the hologram is a phase hologram is proportional to

$$
\tau_{\mathrm{I}}(x, y)=1+\frac{1}{2} \mathrm{e}^{-j \frac{2 \pi}{\lambda_{1}} y\left(\sin \alpha_{r}-\sin \alpha_{o}\right)}+\frac{1}{2} \mathrm{e}^{j \frac{2 \pi}{\lambda_{1}} y\left(\sin \alpha_{r}-\sin \alpha_{o}\right)}
$$

The hologram is illuminated with a polychromatic plane wave with a Gaussian spatial profile, $U_{c}(x, y)$ :

$$
U_{c}(x, y)=\int_{-\infty}^{\infty} u(\lambda) \mathrm{e}^{-\frac{\left(x^{2}+y^{2}\right)}{w^{2}}} \mathrm{e}^{-j \frac{2 \pi}{\lambda} y \sin \alpha_{c}} \mathrm{~d} \lambda
$$

where $u(\lambda)$ is the wave spectrum and $w$ is the half wide of the Gaussian beam.

A wave $U_{i, \mathrm{I}}(x, y)$ emerges from the hologram,

$$
\begin{gathered}
U_{i, \mathrm{I}}(x, y)=U_{C}(x, y) \cdot \tau(x, y)= \\
=\int_{-\infty}^{\infty} u(\lambda) \mathrm{e}^{-\frac{\left(x^{2}+y^{2}\right)}{w^{2}}} \mathrm{e}^{-j \frac{2 \pi}{\lambda} y \sin \alpha_{c}} \mathrm{~d} \lambda+ \\
+\frac{1}{2} \int_{-\infty}^{\infty} u(\lambda) \mathrm{e}^{-\frac{\left(x^{2}+y^{2}\right)}{w^{2}}} \mathrm{e}^{-j\left\{\frac{2 \pi}{\lambda} y\left[\sin \alpha_{c}+\frac{\lambda}{\lambda_{1}}\left(\sin \alpha_{r}-\sin \alpha_{o}\right)\right]\right\}} \mathrm{d} \lambda+ \\
+\frac{1}{2} \int_{-\infty}^{\infty} u(\lambda) \mathrm{e}^{-\frac{\left(x^{2}+y^{2}\right)}{w^{2}}} \mathrm{e}^{j\left\{\frac{2 \pi}{\lambda} y\left[\sin \alpha_{c}+\frac{\lambda}{\lambda_{1}}\left(\sin \alpha_{r}-\sin \alpha_{o}\right)\right]\right\}} \mathrm{d} \lambda= \\
=U_{1, \mathrm{I}}(x, y)+U_{2, \mathrm{I}}(x, y)+U_{3, \mathrm{I}}(x, y)
\end{gathered}
$$

$U_{1, \mathrm{I}}$ is the transmitted order and can be minimized recording the hologram with the suitable phase modulation and illuminating in Bragg condition for the central wavelength of the spectrum. 
$U_{2, \mathrm{I}}$ and $U_{3, \mathrm{I}}$ are +1 and -1 diffracted orders, respectively. In the case of volume holograms, only one of them is diffracted efficiently, depending on the $\alpha_{C}$ value. We suppose that this one is $U_{3, I}$. It is a set of polychromatic plane waves with a Gaussian spatial profile [Fig. 1(a)]. The direction of propagation of each wavelength forms an angle $\alpha_{i}$ with $z$ axis, given by

$$
\sin \alpha_{i}=\sin \alpha_{c}-\frac{\lambda}{\lambda_{1}}\left(\sin \alpha_{r}-\sin \alpha_{o}\right)
$$

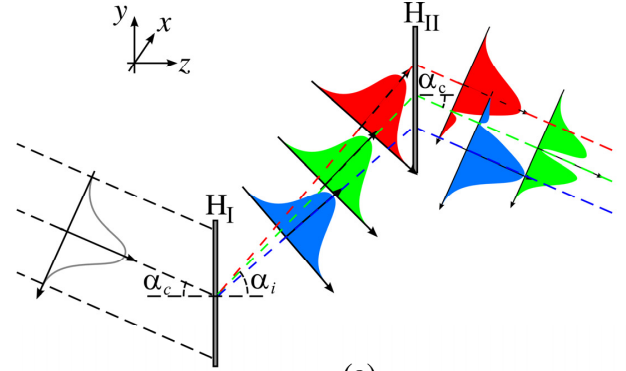

(a)

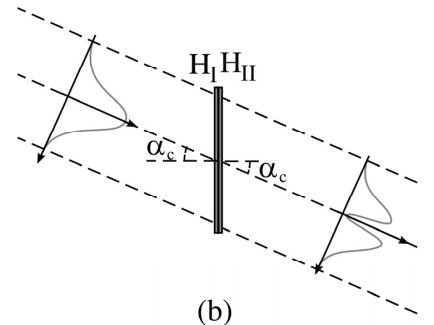

(b)

Fig. 1. Double-grating vortex generator. (a) Separated gratings, (b) Cemented gratings.

We use $U_{3, \mathrm{I}}$ to illuminate a second $\mathrm{VPH}, \mathrm{H}_{\mathrm{II}}$, separated a distance $d$ from $\mathrm{H}_{\mathrm{I}} . \mathrm{H}_{\mathrm{II}}$ is recorded with the interference of a plane wave $U_{r, \mathrm{II}}$ and a vortex beam $U_{o, \text { II }}$,

$$
\begin{gathered}
U_{r, \mathrm{II}}(x, y)=\mathrm{e}^{-j \frac{2 \pi}{\lambda_{1}} y \sin \alpha_{r}} \\
U_{o, \text { II }}(x, y)=\mathrm{e}^{-j\left(\frac{2 \pi}{\lambda_{1}} y \sin \alpha_{0}-m \theta\right)}
\end{gathered}
$$

Where $\theta=\tan ^{-1}(y / x)$ is the angular coordinate at $\mathrm{H}_{\text {II }}$ plane. The complex transmittance recorded is proportional to

$$
\tau_{\text {II }}(x, y)=1+\frac{1}{2} \mathrm{e}^{-j\left(\frac{2 \pi}{\lambda_{1}} y\left(\sin \alpha_{r}-\sin \alpha_{o}\right)+m \theta\right)}+\frac{1}{2} \mathrm{e}^{j\left(\frac{2 \pi}{\lambda_{1}} y\left(\sin \alpha_{r}-\sin \alpha_{o}\right)+m \theta\right)}
$$

The Gaussian profile of $U_{3, \mathrm{I}}$ is kept in the propagation along the distance $d$, but the different wavelenghts are spatially separated and dephased when $U_{3, \mathrm{I}}$ reaches the hologram $\mathrm{H}_{\text {II }}$. A set of vortices emerges from $\mathrm{H}_{\text {II }}$, all with the same topological charge $m$ forming an angle of $\alpha_{c}$ with the $z$-axis. The position of the vortex is the same for all wavelengths, but the Gaussian distribution is spatially shifted for each wavelength, as shown in Fig. 1(a).

If $d=0$, the wave diffracted efficiently by $\mathrm{H}_{\mathrm{II}}$ is given by:

$$
U_{3, \mathrm{II}}(x, y)=\frac{1}{2} \int_{-\infty}^{\infty} u(\lambda) \mathrm{e}^{-\frac{\left(x^{2}+y^{2}\right)}{w^{2}}} \mathrm{e}^{-j\left(\frac{2 \pi}{\lambda} y \sin \alpha_{c}+m \theta\right)} \mathrm{d} \lambda
$$

In this particular case [Fig. 1(b)] the Gaussian distribution is centered on vortex for all wavelengths, that is, $U_{3, \mathrm{II}}$ is an achromatic vortex, without spatial chromatic dispersion and, when it is used with ultrashort laser pulses, without temporal chirp. 


\section{Experimental set-up}

We record $\mathrm{H}_{\mathrm{I}}$ and $\mathrm{H}_{\mathrm{II}}$ with a Coherent Verdi $6 \mathrm{~W} \mathrm{CW}$ laser emitting at $532 \mathrm{~nm}$. We use commercial Slavich PFG-04 dichromated gelatin plates following the process described in [19] to obtain maximum efficiency at $800 \mathrm{~nm}$. The two recording beams forms an angle of $10^{\circ}$, minimum angle required to obtain a volume hologram with the $30 \mu \mathrm{m}$-thick PFG-04 emulsion.

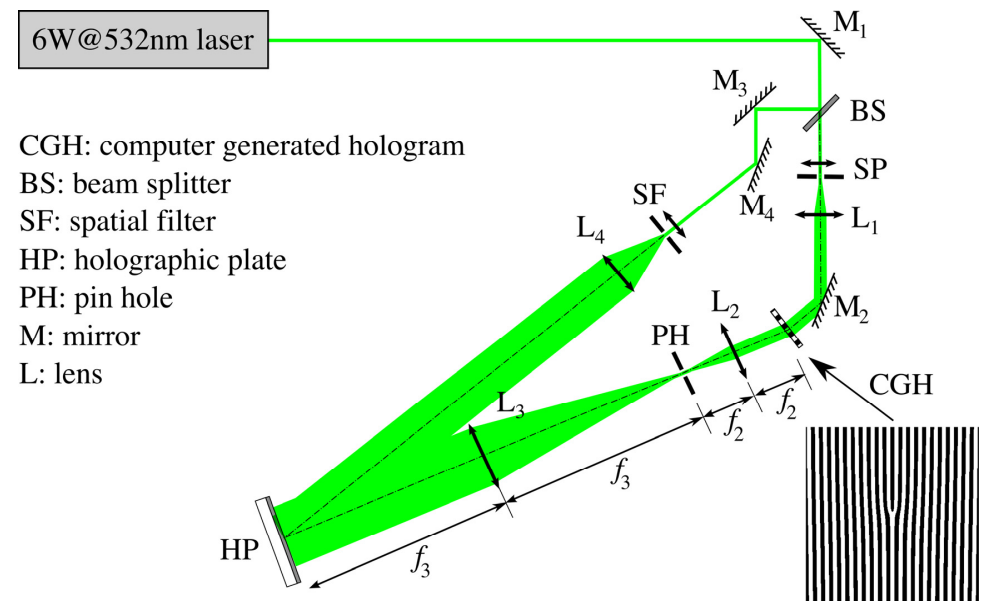

Fig. 2. Recording scheme for a vortex volume hologram.

The recording scheme for $\mathrm{H}_{\mathrm{II}}$ is shown in Fig. 2. The CGH used to generate $U_{o, \mathrm{II}}$ has been made with a three-step process: first, we calculate the pattern given by the interference of $U_{r, \text { II }}$ [Eq. (8)] and $U_{o, \text { II }}$ [Eq. (9)] (when $m=1$ ) with MatLab and print it on paper with a laser writer; second, we photograph the pattern with a reflex camera in Kodak TMax100 film; third, we contact-copy the film pattern onto a PFG-04 dichromated gelatin plate using incoherent light. So we obtain a thin phase CGH with 14 lines $/ \mathrm{mm}$ and $25 \%$ efficiency. The effects of bitmap resolution in the laser printed CGH require one to use spatial filtering techniques to select the desired diffraction order and to avoid bitmap artifacts. We include a 4- $f$ processor to filter the Gaussian optical vortex and to cancel phase propagation terms [15].

The recording scheme for $\mathrm{H}_{\mathrm{I}}$ is similar to that shown in Fig. 2 by replacing the vortex wave with a collimated wave. The angle between beams has to be the same for $\mathrm{H}_{\mathrm{I}}$ and $\mathrm{H}_{\mathrm{II}}$ to guarantee the chromatic compensation.

After processing, $\mathrm{H}_{\mathrm{I}}$ and $\mathrm{H}_{\mathrm{II}}$ are cemented with their emulsions in contact, using Norland NOA61 optical adhesive. This is necessary to avoid changes in the refraction index between holograms and to preserve emulsion from environmental effects.

The maximum efficiency of each grating at Bragg angle for $800 \mathrm{~nm}$ is around $95 \%$, so the sandwich has an efficiency of $90 \%$. Losses of about $15 \%$ are due to reflection on glass surfaces and glass absorption, so absolute efficiency of the compound holographic element is $77 \%$. This efficiency drops when $\lambda$ goes far $800 \mathrm{~nm}$. The dependence of diffractive efficiency with wavelength for the compound element is shown in Fig. 3. Full width al half maximum (FWHM) is $250 \mathrm{~nm}$, and the wavelength range in which the diffractive efficiency is above $80 \%$, is $100 \mathrm{~nm}$. 


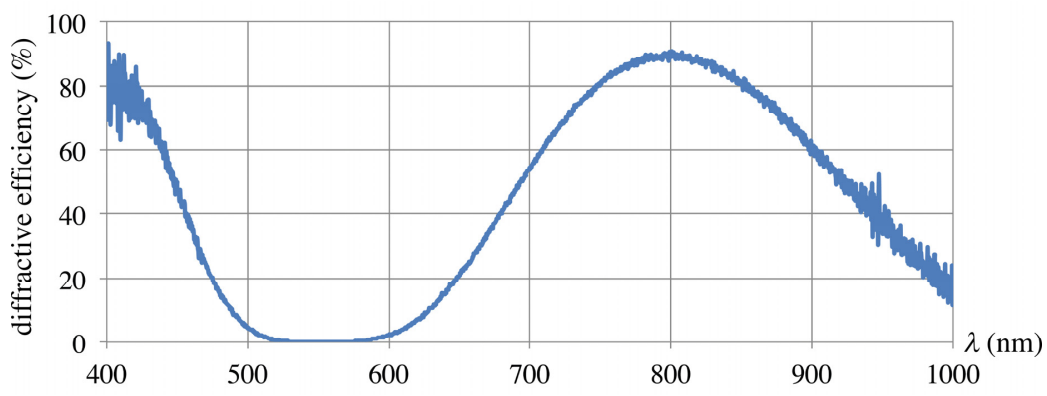

Fig. 3. Diffractive efficiency vs. wavelength for the cemented holographic element.

While diffractive efficiency drops, transmitted light increases, so the transmitted and diffracted light can overlap for some wavelengths. To avoid this effect, we rotate $\mathrm{H}_{\mathrm{I}}$ a small angle around the $z$-axis before join the two holograms. This does not affect the efficiency but causes the transmitted and diffracted beams are separated when they emerge from $\mathrm{H}_{\text {II }}$.

Figure 4(a) shows a $5 \mathrm{~mm}$-wide white light vortex propagated at $1 \mathrm{~m}$ from holographic element. White light illumination is provided by a tungsten light source (Ocean Optics LS-1) with $600 \mathrm{~nm}$ FWHM centered at $850 \mathrm{~nm}$. Figure 4(b) shows interference of diffracted light and transmitted light when the holographic element is illuminated out of Bragg condition. It can be seen the dislocation at the center of the interference. Both figures demonstrate that a broadband achromatic optical vortex has been created.

The holographic material Slavich PFG-04 has high damage threshold [20], so the designed element can be used with ultrashort and ultraintense laser pulses.
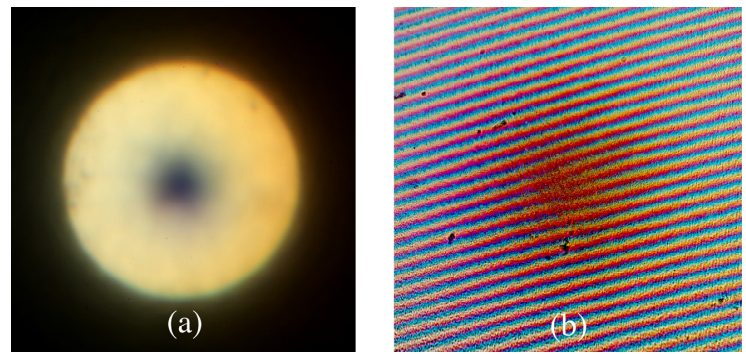

Fig. 4. White light vortex. (a) Propagated at $1 \mathrm{~m}$. (b) Interference with a plane wave.

\section{Conclusions}

We design and construct an element to generate broadband optical vortices with high efficiency by stacking two VPH recorded in Slavich PFG-04 dichromated gelatin emulsion. The angular selectivity of this element is $250 \mathrm{~nm}(\mathrm{FWMH})$ and the absolute efficiency is $77 \%$, but it could be higher if an antireflection coating is used. The solution is compact and easy to align. Although the element constructed in this work has its maximum efficiency at $800 \mathrm{~nm}$, this wavelength can be change during the recording step, so it is possible to construct holographic elements adapted to a particular region of the spectrum.

\section{Acknowledgments}

This research has been supported by the Spanish Ministerio de Economía y Competitividad (grant FIS2012-35433) and the Diputación General de Aragón (Grupo Consolidado TOL, T76) 